\title{
Quantum mechanical description of Stern-Gerlach experiments
}

\author{
G. Potel, ${ }^{1}$ F. Barranco, ${ }^{2}$ S. Cruz-Barrios, ${ }^{3,1}$ and J. Gómez-Camacho ${ }^{1}$ \\ ${ }^{1}$ Departamento de Física Atómica, Molecular y Nuclear, Apartado Postal 1065, 41080 Sevilla, Spain \\ ${ }^{2}$ Departamento de Física Aplicada 3, E.S.I. Isla de la Cartuja, Sevilla, Spain \\ ${ }^{3}$ Departamento de Física Aplicada 1, E.U.P. Virgen de África, Sevilla, Spain
}

(Received 21 September 2004; published 27 May 2005)

\begin{abstract}
The motion of neutral particles with magnetic moments in an inhomogeneous magnetic field is described in a quantum mechanical framework. The validity of the semiclassical approximations which are generally used to describe these phenomena is discussed. Approximate expressions for the evolution operator are derived and compared to the exact calculations. Focusing and spin-flip phenomena are predicted. The reliability of SternGerlach experiments to measure spin projections is assessed in this framework.
\end{abstract}

DOI: 10.1103/PhysRevA.71.052106

\section{INTRODUCTION}

The Stern-Gerlach experiment consists in taking a beam of particles that have a neutral electric charge, but a finite magnetic moment, and passing them through an inhomogeneous magnetic field. The observed result is that the particles deflect differently depending on the spin projection along the magnetic field. So, by measuring the deflection, one can infer the value of the spin projection of the particles along the direction of the magnetic field. The description of this phenomenon is done with the following assumptions.

(i) The spin projection along the $z$ axis, taken along the magnetic field at the center of the beam, is conserved.

(ii) Particles with different spin projections along the $z$ axis, as they go through the inhomogeneous magnetic field, suffer a force in the $z$ direction that is given by the product of the magnetic moment times the gradient of the field times the spin projection.

This is what we will call the textbook description of the Stern-Gerlach experiment [1-4]. Thus, considering the particle position as a pointer and the spin projection as the quantum property to be measured, the Stern-Gerlach setup is associated with a measurement operator on the spin state which has as eigenvalues the spin projections along the $z$ axis. Under the textbook description, the Stern-Gerlach experiment corresponds to an "ideal" measurement, in the sense of von Neumann [5], because the quantum state is not modified by the measurement process when it is an eigenstate of the measuring apparatus. Besides, it is "completely reliable," in the sense discussed in [6], because the position is completely correlated with the spin projection.

However, when the experiment is investigated in more detail, the situation becomes more complicated. As the magnetic field has zero divergence, then it is not possible to have a gradient of the field only in one direction. This produces terms in the Hamiltonian that can change the spin of the incident particle. A detailed investigation of these effects was made in a recent publication [7], making use of the concept of coherent internal states [8] in a semiclassical approach. In this approach, it is shown that the quantum mechanical wave function which describes the motion of a system with internal degrees of freedom can be approximated by a single trajectory only for certain internal states which are called co- herent internal states. These internal states evolve in time according to an evolution operator which is determined by the interaction evaluated along the trajectory. The coherent internal states, in the case of the Stern-Gerlach experiments, are states with definite projection along the direction of the magnetic field. This direction may vary depending on the position of the particle, because the magnetic field is not homogeneous.

The main result of [7] is that, indeed, when a beam of particles goes through a Stern-Gerlach magnet, the different spin projections deviate depending on the spin projection. However, when the size of the beam is not very small compared to the range of inhomogeneity of the magnetic field, additional effects occur.

(i) There is a focusing effect, so that the particles deviating in the direction in which the field decreases tend to focus, while those going in the direction of increasing field tend to defocus.

(ii) There are some particles with a given spin projection which deviate as those with a different spin projection. So the Stern-Gerlach setup is not, even in theory, a "completely reliable" measuring apparatus.

(iii) There are some particles, with a definite spin projection along the quantization axis, which change the spin projection as they go through the magnet. So the Stern-Gerlach setup is not an "ideal" measurement apparatus, as successive measurements will not give exactly the same results.

This is what we will call the semiclassical description of the Stern-Gerlach experiment. Note that if we associate the particle position after the magnet as a "pointer," which gives the result of the measurement of the spin projection along the $z$ axis, then we conclude that, in the semiclassical description, the Stern-Gerlach experiment is not an ideal measurement, because it can alter the spin projection, or a completely reliable one, because the position is not always correlated with the spin projection.

These conclusions were obtained in a semiclassical framework, in which the motion of the particles was described by classical trajectories which depended in the spin projection along the magnetic field that they encountered. Our motivation here is to see whether the same conclusions hold when the full quantum mechanical problem is considered. In Sec. II we formulate the time-dependent quantum 
mechanical problem of a wave packet going through a SternGerlach magnet and discuss the validity of the textbook and semiclassical approaches. In Sec. III we present the numerical solution of the quantum mechanical problem. In Sec. IV we investigate several analytic approximations to the problem, considering the validity of the concept of coherent internal states. In Sec. V we discuss the interpretation of SternGerlach experiments as measurements devices. Sec. VI is for a summary and conclusions.

\section{QUANTUM MECHANICAL FORMULATION}

We want to investigate the effect of an inhomogeneous magnetic field on the evolution of a quantum wave packet. The situation that we will consider is a magnetic field that has components in the $X$ and $Z$ directions, but not in the $Y$ direction. This magnetic field has a length $L$, and it can be written as

$$
\vec{B}=\left(B_{0}+B_{1} Z\right) \vec{u}_{z}-B_{1} X \vec{u}_{x}, \quad 0 \leqslant Y \leqslant L .
$$

We use the capital letters $X, Y, Z, T$ to represent magnitudes with dimensions. Lowercase $x, y, z, t$ correspond to dimensionless quantities. We neglect border effects around $Y=0$ or $Y=L$. Note that this field fulfills $\nabla \vec{B}=0$ and also $\nabla \times \vec{B}=0$, as should be expected for a magnetic field in the region where there are no currents. These conditions were not fulfilled in the case discussed in textbooks such as [1-3].

The Hamiltonian which describes a nonrelativistic neutral particle which enters in this field is given by

$$
\mathcal{H}=\frac{P_{X}^{2}+P_{Y}^{2}+P_{Z}^{2}}{2 M}-\mu \vec{B} \cdot \vec{I}
$$

where $\mu$ is the magnetic moment and $\vec{I}$ is the spin operator.

We consider now a wave packet $\left|\Psi(T) ; m_{0}\right\rangle$ which enters into this field. Initially, the wave packet can be characterized in coordinate space as a Gaussian which is moving in the $y$ direction, while the initial spin projection along the $Z$ axis is $m_{0}$ :

$$
\begin{aligned}
\langle X Y Z, m| \Psi(T= & \left.0) ; m_{0}\right\rangle=N \exp \left(-\frac{X^{2}+Y^{2}+Z^{2}}{2 \sigma^{2}}\right) \\
& \times \exp \left(i k_{y} Y\right) \delta\left(m, m_{0}\right) .
\end{aligned}
$$

Note that, neglecting the effects of the border, the $Y$ component of the wave function is not affected by the interaction. Border effects will be relevant when the transit time, which is the time that the particle takes to go from the situation in which the field vanishes $(\vec{B}=0)$ to the situation where the field is stationary $\left(\vec{B} \simeq B_{0} \vec{u}_{z}\right)$, is short compared to the precession time of the spin in the magnetic field. An estimate of the transit time is $t_{t}=B_{0} /\left(B_{1} v\right)$. The precession time is $t_{p}$ $=\hbar /\left(B_{0} \mu\right)$. It can be seen that, for realistic cases, the transit time, although short compared to the time that the beam spend within the magnet, is always larger than the precession time. So the spins of the particles of the beam have the time to adapt to the magnetic field in which they enter.

The wave function is given by a wave packet that can be factorized into a $Y$ component and an $(X, Z)$ component. The
$Y$ component will evolve freely inside the magnet, because the Hamiltonian does not have any interaction term which depends on $Y$, once that the border effects (occurring at $Y$ $=0$ and $Y=L$ ) are found to be negligible.

Note that the wave packet will stay within the magnetic field during a time $\tau=L / v_{y}$, where $v_{y}=\hbar k_{y} / M$. Assuming that the size of the wave packet $\sigma$ is very small compared to $L$ and taking into account that the transit time $t_{t}$ is much smaller than $T$, we can consider that the magnetic field starts at $T=0$ and finishes at $T=\tau$. So we focus on solving the two-dimensional time-dependent problem, which corresponds to calculating the time evolution between the time $T=0$ and $T=\tau$ in a Hamiltonian

$$
H=\frac{P_{X}^{2}+P_{Z}^{2}}{2 M}-\mu \vec{B} \cdot \vec{I},
$$

considering that the initial wave function is

$$
\left\langle X Z ; m \mid \Phi(T=0) ; m_{0}\right\rangle=N \exp \left(-\frac{X^{2}+Z^{2}}{2 \sigma^{2}}\right) \delta\left(m, m_{0}\right) .
$$

It is convenient to make use of dimensionless variables. So we define $x=X / \sigma, z=Z / \sigma, t=T / \tau$, and $h=H \tau / \hbar$. Then, the equation of motion becomes

$$
h\left|\Phi(t) ; m_{0}\right\rangle=i \frac{d}{d t}\left|\Phi(t) ; m_{0}\right\rangle .
$$

The dimensionless Hamiltonian can be written as $h=h_{0}+v$, with

$$
h_{0}=\frac{A}{2}\left(p_{x}^{2}+p_{z}^{2}\right), \quad v=-S\left[I_{z}\left(z+z_{0}\right)-I_{x} x\right]
$$

where $p_{x}=-i d / d x, p_{z}=-i d / d z$, and the dimensionless parameters $A, S, z_{0}$ are

$$
A=\frac{\hbar \tau}{M \sigma^{2}}, \quad S=\frac{\mu B_{1} \tau \sigma}{\hbar}, \quad z_{0}=\frac{B_{0}}{\sigma B_{1}} .
$$

The adiabaticity parameter $A$ is the ratio of the interaction time $\tau$ to the natural time of expansion of the Gaussian packet. The separation parameter $S$ is the ratio of the momentum change induced by the magnetic field gradient divided by the momentum width of the Gaussian packet. The inhomogeneity parameter $z_{0}$ determines the relative change of the magnetic field in the range of the Gaussian. Note that in the position $\left(x=0, z=-z_{0}\right)$, the magnetic field vanishes. Note that the product $A S=\mu B_{1} \tau^{2} / M \sigma$ is independent of $\hbar$. This magnitude is related to the deviation of the beam in the magnet. For a given trajectory, which is determined by a fixed value of the product $A S$, the classical limit is reached as $S \rightarrow \infty$ and $A \rightarrow 0$. Note that this corresponds to making $\hbar \rightarrow 0$ in Eqs. (8).

\section{Validity of the semiclassical descriptions}

We will now discuss the validity of the semiclassical and textbook descriptions of the Stern-Gerlach experiment. It should be noticed that, in general, a beam of particles is not given by a pure quantum mechanical state, but rather by a 
mixture of small quantum wave packets. For definiteness, we consider that initially one has a distribution of particles described as a Gaussian mixture, of range $\sigma_{m}$, of small Gaussian wave packets of range $\sigma$. The beam profile will then be characterized by a Gaussian of range $\sigma_{t}=\sqrt{\sigma_{m}^{2}+\sigma^{2}}$. The conditions required, in order to justify the semiclassical description done in [7] are the following.

(a) The inhomogeneity of the magnetic field over the quantum size of the wave packet should be small: $\sigma B_{1} \ll B_{0}$. This implies that $z_{0} \gg 1$.

(b) The momentum change should be large compared to the quantum spread of the beam momentum: $\mu B_{1} \tau \gg \hbar / \sigma$. This implies that $S \gg 1$.

Note that these conditions are very well satisfied in realistic situations for Stern-Gerlach experiments. However, the validity of the textbook description requires also the far more stringent condition $\sigma_{t} B_{1} \ll B_{0}$, which requires a very strong field $B_{0}$ or, alternatively, a very thin beam.

The purpose of this work is to investigate the full quantum solution of this problem for values of the parameters $z_{0}$ and $S$ which are not necessarily very large, so that the semiclassical and textbook descriptions become dubious. Nevertheless, in order to have a reference to compare the quantum calculation, we recall the expected results in the textbook description. The trajectory of the center of the wave packet inside the magnet is given by the expression

$$
z_{m}(t)=1 / 2(S A) m t^{2},
$$

which depends on the spin projection $m$. Note that, after the interaction $(t=1)$, the positions of the center of the wave packets for each spin projection are given by $z_{m}(1)$ $=S A m / 2$ and their velocities are $\dot{z}_{m}(1)=S A m / 2$. If, after the interaction, the beam evolves freely during a time $t_{d}$, then the positions of the center of the wave packets are expected to be given by

$$
z_{m}\left(t_{d}\right)=\left(1 / 2+t_{d}\right)(S A) m .
$$

As a typical value of the drift time $t_{d}$ we will consider the time necessary to reach the position $z_{m}=-z_{0}$, for the spin projection $m=-1 / 2$,

$$
t_{d}=2 z_{0} /(S A)-1 / 2 .
$$

Thus we would expect that, after a drift time $t_{d}$, particles with spin projection $m=1 / 2$ should appear around $z=z_{0}, x$ $=0$, and particles with spin projection $m=-1 / 2$ should appear around $z=-z_{0}, x=0$.

\section{NUMERICAL CALCULATIONS}

We consider the scattering of a spin- $1 / 2$ particle. We expand the wave function into two components, which have definite spin projections along the $z$ axis,

$$
\begin{gathered}
\left\langle x z ; m=1 / 2 \mid \Phi(t) ; m_{0}\right\rangle=\alpha(x, z, t) e^{i t S z_{0} / 2}, \\
\left\langle x z ; m=-1 / 2 \mid \Phi(t) ; m_{0}\right\rangle=\beta(x, z, t) e^{-i t S z_{0} / 2},
\end{gathered}
$$

and the Schrödinger equation for the $(x, z)$ plane can be written as
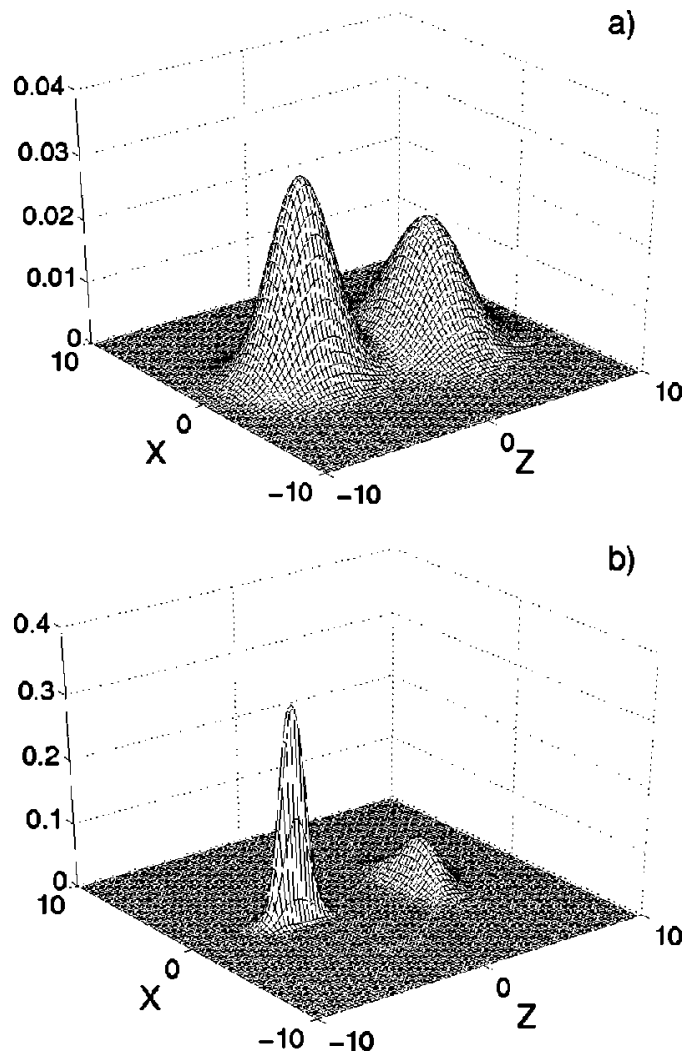

FIG. 1. Probability distribution for an unpolarized wave packet after going through an inhomogeneous magnetic field. Note the focusing effect of the lower component, which corresponds predominantly to $m=-1 / 2$. The upper figure corresponds to $A=0.5$, $S=4$. The lower figure is for $A=0.1, S=20$, which is closer to the classical limit.

$$
\begin{aligned}
& {\left[\begin{array}{cc}
\frac{A}{2}\left(p_{x}^{2}+p_{z}^{2}\right)-\frac{S}{2} z & \frac{S}{2} x \\
\frac{S}{2} x & \frac{A}{2}\left(p_{x}^{2}+p_{z}^{2}\right)+\frac{S}{2} z
\end{array}\right]\left[\begin{array}{l}
\alpha(x, z, t) \\
\beta(x, z, t)
\end{array}\right]} \\
& \quad i \frac{d}{d t}\left[\begin{array}{l}
\alpha(x, z, t) \\
\beta(x, z, t)
\end{array}\right],
\end{aligned}
$$

where $\alpha(x, z, t)$ and $\beta(x, z, t)$ are the components of the spinor in the basis of the eigenstates of $I_{z}$. The numerical solution of this equation has already been performed by Garraway and Stenholm [9]. However, they considered the case in which $z_{0}$ was large, so their numerical result corresponded to the textbook interpretation. A similar problem has been addressed by Franca et al. [10], but they made use of the adiabatic approximation, neglecting the kinetic energy during the interaction time.

To follow our approach we must first write both components of the spinor as linear combinations of harmonic oscillator functions, so that

$$
\alpha(x, z, t)=\sum_{n m} a_{n m}(t) \phi_{n}(x) \phi_{m}(z)
$$




$$
\beta(x, z, t)=\sum_{n m} b_{n m}(t) \phi_{n}(x) \phi_{m}(z),
$$

where $\phi_{n}(x)$ and $\phi_{m}(z)$ are the harmonic oscillator eigenstates of order $n$ and $m$ in the $x$ and $z$ directions, respectively. To calculate the time-dependent coefficients $a_{n m}(t)$ and $b_{n m}(t)$ of the expansion, it is natural to rewrite Eq. (13) in terms of the well-known creation and destruction operators

$$
a_{x}=\frac{1}{\sqrt{2}}\left(x+i p_{x}\right), \quad a_{x}^{\dagger}=\frac{1}{\sqrt{2}}\left(x-i p_{x}\right),
$$

Thus, substituting the operators (15) into Eq. (13), we obtain the desired system of ordinary coupled differential equations for the coefficients of the expansion of $\alpha(x, z, t)$ and $\beta(x, z, t)$ :

$$
\begin{aligned}
\dot{a}_{n m}= & i \frac{A}{4}\left[a_{n+2, m} \sqrt{(n+1)(n+2)}+a_{n-2, m} \sqrt{n(n-1)}+a_{n, m+2} \sqrt{(m+1)(m+2)}+a_{n, m-2} \sqrt{m(m-1)}-2 a_{n m}(n+m+1)\right] \\
& +i \frac{S}{2 \sqrt{2}}\left[a_{n, m+1} \sqrt{m+1}+a_{n, m-1} \sqrt{m}-\left(b_{n+1, m} \sqrt{n+1}+b_{n-1, m} \sqrt{n}\right) e^{-i S z_{0} t}\right], \\
\dot{b}_{n m}= & i \frac{A}{4}\left[b_{n+2, m} \sqrt{(n+1)(n+2)}+b_{n-2, m} \sqrt{n(n-1)}+b_{n, m+2} \sqrt{(m+1)(m+2)}+b_{n, m-2} \sqrt{m(m-1)}-2 b_{n m}(n+m+1)\right] \\
& +i \frac{S}{2 \sqrt{2}}\left[-b_{n, m+1} \sqrt{m+1}-b_{n, m-1} \sqrt{m}-\left(a_{n+1, m} \sqrt{n+1}+a_{n-1, m} \sqrt{n}\right) e^{i S z_{0} t}\right],
\end{aligned}
$$

where the overdot stands for differentiation with respect to the dimensionless parameter $t$. This system is solved using a fourth-order Runge-Kutta method. The number of harmonic oscillator basis functions needed in the calculation was typically of the order of 40 in each coordinate.

We have performed calculations using typical values of $A=0.5, S=4$, and $z_{0}=4$. This corresponds to a case in which the magnetic field vanishes at a distance of $4 \sigma$. The time of the interaction is such that the width of the beam would increase by a factor of $\sqrt{1+A^{2}}$. The magnetic field gradient is such that each component of the magnetic field will acquire a momentum of $S \hbar / 2 \sigma$, in opposite directions. As a comparison, we have also considered calculations with $A=0.1, S$ $=20$, and $z_{0}=4$, which produce the same deviation of the beam, but are closer to the classical limit.

After the interaction, we consider a drift time $t_{d}$, given by Eq. (11), during which the system evolves in the free Hamiltonian, so that the center of the $m= \pm 1 / 2$ wave packet would reach the point $z= \pm z_{0}$, according to the textbook description.

In Fig. 1 we represent the probability distribution of a wave packet, corresponding initially to an unpolarized beam. This is given by

$$
P_{0}(x, z)=\frac{1}{2} \sum_{m m_{0}}\left|\left\langle x, z ; m \mid \Phi(t) ; m_{0}\right\rangle\right|^{2} .
$$

The focusing effect can be clearly seen by comparing the shape of the distributions for the upper and lower components, which correspond predominantly to $m=1 / 2$ and $m$ $=-1 / 2$, respectively. The effect of the focusing is increased as $A$ decreases and $S$ increases. So we have confirmed that the focusing effect that was predicted in the semiclassical calculation in [8] is a genuine result that appears in the quantum mechanical calculation, although it is diffused if the adiabaticity parameter $A$ has a sizable value. It should be noticed that this focusing effect was also found in the calculations presented in [9].

In contrast to the textbook description, even if the initial beam has a definite spin projection along the $z$ axis, after the scattering process this spin projection can change. We have evaluated the probability that the particles change their spin projection along the $z$ axis. It should be noticed that the probability of going from spin up to spin down is not exactly the same as that of going from spin down to spin up. For the reference case $\left(A=0.5, S=4, z_{0}=4\right)$, we obtain that $p(1 / 2$, $-1 / 2)=0.0166$ and $p(-1 / 2,1 / 2)=0.0198$.

The spin-flip phenomenon also appears in the semiclassical description, because not all the particles that compose the beam see the magnetic field along the $z$ axis. The semiclassical spin-flip probability is $p(1 / 2,-1 / 2)=p(-1 / 2,1 / 2)$ $=0.0156$, which depends only on the value of $z_{0}$. This is in good qualitative agreement with the quantum calculations. In Fig. 2 we represent the spatial distribution of the spin-flip probability. Note that the spin-flip probability vanishes for particles coming out along the $z$ axis. The spatial distribution of the spin-flip probability is in qualitative agreement with the semiclassical calculation, which becomes more accurate as one makes the limit $A \rightarrow 0, S \rightarrow \infty$, with $A S$ constant.

The results of our calculations can be summarized as follows: When a beam of particles, described by a Gaussian 


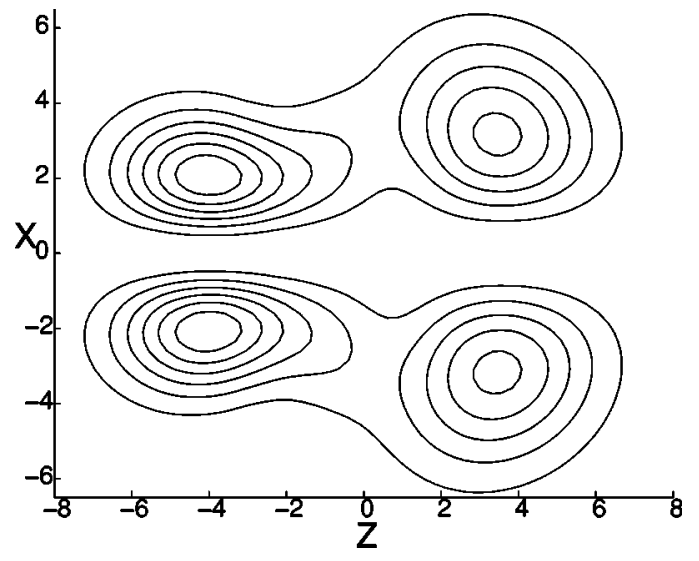

FIG. 2. Contour plot of the probability distribution of the spinflip component (spin up to spin down) of the wave function. The maximum is $3.3 \times 10^{-4}$.

wave function and with a given spin projection along the $z$ axis, goes through an inhomogeneous magnetic field, most of the particles scatter as expected in the textbook description. However, a sizable fraction of them, which depends on $z_{0}$ (about $2 \%$ for $z_{0}=4$ ), suffer a change of the spin projection (spin flip). From these particles that suffer spin flip, about half scatter in the same direction as the majority of the particles and the other half scatter in the opposite direction. We can conclude that the spin-flip effect described in the semiclassical description, which was not present in the textbook description of Stern-Gerlach experiments, is supported by the full quantum mechanical calculations. Also, we confirm that the Stern-Gerlach experiment, when considered as a measurement apparatus of the spin projection, is not an ideal measurement (because there is spin flip) and it is not fully reliable (because there is not an exact correlation between the initial spin projection and the final position of the particle).

However, there are qualitative features of the full quantum mechanical result, such as the difference between updown and down-up spin-flip probabilities, that are not present in the semiclassical description and require further investigation.

\section{APPROXIMATE TREATMENTS}

Having solved numerically the problem, we will consider several approximate treatments to improve our understanding of the phenomena under consideration. The starting point is the exact evolution operator and the free evolution operator

$$
U(t)=\exp \left[-i\left(h_{0}+v\right) t\right], \quad U_{0}(t)=\exp \left(-i h_{0} t\right) .
$$

It should be noticed that $h_{0}$ and $v$ do not commute. Thus, $a$ priori there is not a single basis of spin states where the evolution operator is diagonal. Nevertheless, it can be argued that the interaction $v$ dominates over the free Hamiltonian $h_{0}$. That would indicate that the eigenstates of $v$, which are states with definite spin projection along the magnetic field (and hence coherent internal states), should play an impor- tant role in the approximate solution of this problem. In this section we derive several approximate expressions which make use of expansions of the exact evolution operator in terms of $v$ and $h_{0}$ and its commutators.

We can use the coordinates

$$
\rho=\sqrt{\left(z+z_{0}\right)^{2}+x^{2}}, \quad \beta=\arctan \frac{x}{\left(z+z_{0}\right)},
$$

and refer the spin components to the direction of the magnetic field at each position:

$$
I_{B}=I_{z} \cos (\beta)-I_{x} \sin (\beta), \quad I_{T}=I_{z} \sin (\beta)+I_{x} \cos (\beta) .
$$

In terms of these variables, the initial state can be expressed as

$$
\begin{aligned}
\langle\rho \beta ; m| \Phi(t & \left.=0) ; m_{0}\right\rangle \\
& =N \exp \left(-\frac{\rho^{2}-2 \rho z_{0} \cos \beta+z_{0}^{2}}{2}\right) \delta\left(m, m_{0}\right)
\end{aligned}
$$

and $h_{0}$ and $v$ take the expressions

$$
h_{0}=\frac{A}{2}\left(p_{\rho}^{2}+\rho^{-2} p_{\beta}^{2}\right), \quad v=-S \rho I_{B},
$$

where $p_{\rho}$ and $p_{\beta}$ are the momenta associated with $\rho$ and $\beta$. The relevant commutators are the following:

$$
\begin{gathered}
{\left[h_{0}, v\right]=i A S\left(p_{\rho} I_{B}-\left\{p_{\beta}, I_{T}\right\} / 2 \rho\right),} \\
{\left[\left[h_{0}, v\right], v\right]=-A S^{2}\left(I_{B}^{2}+I_{T}^{2}-\left\{p_{\beta}, I_{y}\right\} / 2 .\right.}
\end{gathered}
$$

Note that $\left[\left[h_{0}, v\right], h_{0}\right]=0$ and $\left[\left[\left[h_{0}, v\right], v\right], h_{0}\right]=0$. For spin$1 / 2$ particles, $I=1 / 2, I_{B}^{2}=I_{T}^{2}=1 / 4$.

\section{A. Adiabatic approximation}

The simplest approximation for the evolution operator consists in neglecting completely the effect of $h_{0}$. This leads to the adiabatic approximation, given by

$$
U(t) \simeq \exp (-i t v)=\exp \left(i t S \rho I_{B}\right) .
$$

Note that this expression conserves the projection of the spin along the direction of the magnetic field. Thus, it is convenient to expand the initial spin state into states $|n\rangle$ which fulfill $I_{B}|n\rangle=n|n\rangle$. This can be done considering the rotation of an angle $\beta$ around the $y$ axis which takes the $z$ axis to the direction of the magnetic field. Thus, the adiabatic expression for the wave function after the interaction becomes

$$
\begin{aligned}
\left\langle\rho \beta ; m \mid \Phi(t) ; m_{0}\right\rangle= & N \exp \left(-\frac{\rho^{2}-2 \rho z_{0} \cos \beta+z_{0}^{2}}{2}\right) \\
& \times \sum_{n} d_{n m}^{1 / 2}(\beta) \exp (\text { in } \rho S t) d_{n m_{0}}^{1 / 2}(\beta) .
\end{aligned}
$$

Note that this expression is equivalent to Eq. (3.3) in [10], 
where they expanded the wave function in components that had definite spin projections along the local magnetic field. This expression contains the qualitative features described in the numerical calculation. There is a spin-flip probability, as $m \neq m_{0}$. The focusing effect appears when this adiabatic wave function undergoes a free evolution during a time $t_{d}$ after the interaction. However, during the interaction time, the probability distribution is frozen.

\section{B. Pseudoadiabatic approximation}

The next approximation consists in neglecting the commutator $\left[h_{0}, v\right]$. This leads to the pseudoadiabatic approximation, given by

$$
U(t) \simeq \exp (-i t v) \exp \left(-i t h_{0}\right)=\exp \left(i t S \rho I_{B}\right) U_{0}(t) .
$$

This expression also conserves the projection of the spin along the direction of the magnetic field, but starting from a wave function that has evolved freely during the interaction time $t$. The wave function has an analytic expression given by

$$
\begin{aligned}
\left\langle\rho \beta ; m \mid \Phi(t) ; m_{0}\right\rangle= & N \exp \left(-\frac{\rho^{2}-2 \rho z_{0} \cos \beta+z_{0}^{2}}{2(1+i A t)}\right) \\
& \times \sum_{n} d_{n m}^{1 / 2}(\beta) \exp (i n \rho S t) d_{n m_{0}}^{1 / 2}(\beta) .
\end{aligned}
$$

The difference of this expression with the adiabatic one lies in the fact that the Gaussian wave packet gets wider during the interaction time, by a factor $\sqrt{1+A^{2}}$, which is the widening of the free wave packet during the interaction time.

\section{Coherent-state approximation}

We consider the expansion of the evolution operator up to the third order commutator. The following relations can be derived:

$$
\begin{aligned}
U(t) \simeq & \left.\exp \left(\frac{(-i t)^{3}}{6}\left[\left[h_{0}, v\right], v\right]\right]\right) \exp (-i t v) \\
& \times \exp \left(\frac{(-i t)^{2}}{2}\left[h_{0}, v\right]\right) U_{0}(t) .
\end{aligned}
$$

This expression is the basis for an analytic treatment of the wave function. For that purpose, we note that the dominant terms in the evolution operator are those which conserve the spin projection along the direction of the magnetic field. The strongly oscillating factor $\exp (-i t v)$ tends to cancel the terms that do not conserve $I_{B}$. We retain in the expansion only those terms which commute with $I_{B}$. This leads to the expression

$$
U(t) \simeq \exp \left(-i t^{3} A S^{2} / 12\right) \exp \left(i t S \rho I_{B}\right) \exp \left(-i t^{2} A S p_{\rho} I_{B}\right) U_{0}(t) .
$$

The operator $\exp \left(-i t^{2} A S p_{\rho} I_{B}\right)$, when acting on eigenstates of $I_{B}$, generates a displacement in $\rho$, which is given by $\rho_{f}=\rho_{i}$ $+t^{2} A S I_{B}$. This leads to an analytic expression for the wave function, given by

$$
\begin{aligned}
& \left\langle\rho \beta ; m \mid \Phi(t), m_{0}\right\rangle \\
& \quad=\exp \left(i A S^{2} t^{3} / 12\right) N \sum_{n} d_{n m}^{1 / 2}(\beta) \exp (i n \rho S t) \sqrt{\frac{\rho_{n}}{\rho}} \\
& \quad \times \exp \left(-\frac{\rho_{n}^{2}-2 \rho_{n} z_{0} \cos \beta-z_{0}^{2}}{2(1+i A t)}\right) d_{n m_{0}}^{1 / 2}(\beta),
\end{aligned}
$$

where $\rho_{n}=\rho-n A S t^{2} / 2$. This wave function conserves the spin projection along the direction of the magnetic field. Thus, the states with a definite spin projection along the magnetic field in each position correspond to the coherent internal states introduced in Ref. [7]. So we call this approximation the coherent-state approximation. Note that in this approximation the wave function not only gets wider during the interacting region, but the components with different values of $I_{B}$ separate.

\section{Symmetrized approximation}

We can approximate the evolution operator by the following expression, which is correct up to commutators of fourth order:

$$
U(t) \simeq U_{0}(t / 2) \exp \left\{-i t v-(-i t)^{3}\left[\left[h_{0}, v\right], v\right] / 12\right\} U_{0}(t / 2) .
$$

Neglecting the terms that do not commute with $I_{B}$, we have

$$
U(t) \simeq \exp \left(i t^{3} A S^{2} / 24\right) U_{0}(t / 2) \exp \left(i t S \rho I_{B}\right) U_{0}(t / 2) .
$$

The wave function can be written as

$$
\left|\Phi(t) ; m_{0}\right\rangle=\exp \left(i A S^{2} t^{3} / 24\right) U_{0}(t / 2)\left|\Phi^{\prime}(t) ; m_{0}\right\rangle,
$$

where

$$
\begin{aligned}
\left\langle\rho \beta ; n \mid \Phi^{\prime}(t) ; m_{0}\right\rangle= & N \exp \left(-\frac{\rho^{2}-2 \rho z_{0} \cos \beta+z_{0}^{2}}{2(1+i A t / 2)}\right) \\
& \times \sum_{n} d_{n m}^{1 / 2}(\beta) \exp (-i n \rho S t) d_{n m_{0}}^{1 / 2}(\beta),
\end{aligned}
$$

which, although it is not completely analytic, it can be applied to evaluate the expansion of the wave function in a harmonic oscillator basis. This approximation corresponds to split the effect of $U_{0}(t)$ during the interaction symmetrically, taking half of it before and half of it after the interaction. Note that here also the evolution associated with the interaction conserves the spin projection along the magnetic field. We call this the symmetrized approximation.

\section{E. Comparison with the exact calculation}

We have performed calculations with all the approximations. We find that the qualitative characteristics of the exact calculations discussed above, which are the focusing effect in the component which goes to negative $z$ values and the 


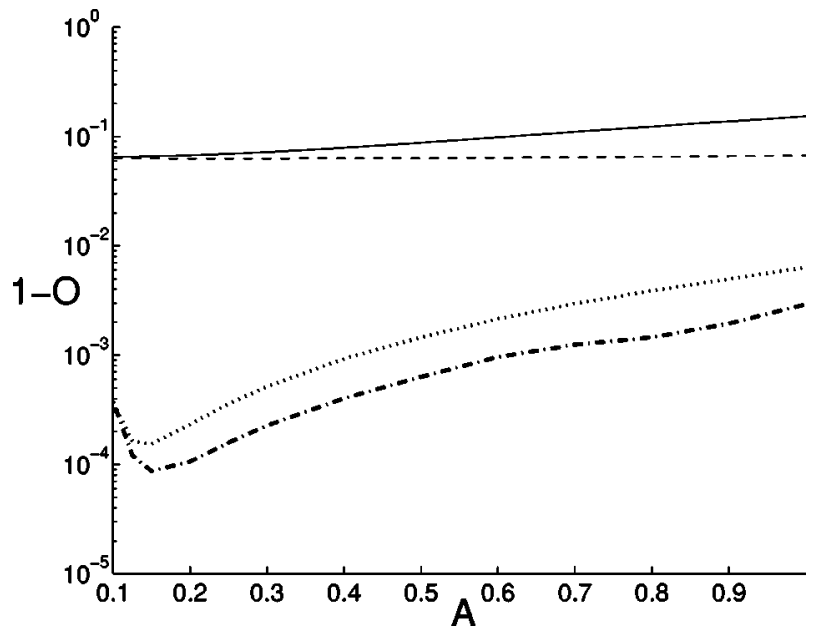

FIG. 3. Overlaps of the approximate wave functions with the exact one, as a function of the adiabaticity parameter, for $S A=2$. The value $1-O=0$ correspond to perfect agreement. The solid line is the adiabatic approximation, the dashed line is the pseudoadiabatic approximation, the dotted line is the coherent-state approximation, and the dot-dashed line is the symmetrized approximation.

presence of spin-flip components, appear in all the calculations. The quantitative differences between the different approaches arise in the momentum distribution of the spin flip component. This comes out symmetric in the adiabatic and pseudoadiabatic approximations (same probability distribution for positive and negative momenta) and not fully symmetric in the coherent-state or symmetrized approximations, in closer agreement with the exact calculations.

To evaluate the quality of these approximations, we have calculated the average of the overlap between the exact and approximate calculations. This overlap is defined as

$$
O=\frac{1}{2}\left|\sum_{m_{0}}\left\langle\Phi_{\mathrm{ex}}(t=1) ; m_{0} \mid \Phi_{\mathrm{ap}}(t=1) ; m_{0}\right\rangle\right| .
$$

They are displayed in Fig. 3, as a function of the adiabaticity parameter $A$, for a fixed value of the product $A S=2$, which determines the deviation of the center of the wave packet in the magnetic field, as shown in Eq. (9). The quantity $1-O$ is about $10 \%$ for a wide range of values of $A$. In particular, for $A=0.5$ and $S=4,1-0=0.088$ for the adiabatic calculation and $1-O=0.064$ for the pseudoadiabatic calculation. On the contrary, the symmetrized and coherent-state approximations are much better, so that $1-O$ is about $0.1 \%$. In particular, for $A=0.5$ and $S=4,1-0=0.0015$ for the coherent-state and $1-0=0.0006$ for the symmetrized calculations. The reason for this better agreement arises from the fact that the coherent-state and symmetrized calculations allow for the distortion in the wave function produced by the magnetic field gradient, while for the adiabatic and pseudoadiabatic calculations the effect of the field contributes only to a phase.

In all the calculations that we have performed, the quality of the approximated calculations improves as one goes from the adiabatic to the pseudoadiabatic to the coherent state and finally to the symmetrized approximations. Globally considered, the approximations deteriorate as the product $S A$ gets larger, because then there is more distortion introduced in the wave function due to the combined effect of the interaction and the free Hamiltonian.

A very interesting case is the limit $A \rightarrow 0, S \rightarrow \infty$ for fixed values of $A S$. Naively, one would expect that the adiabatic approximation would be adequate here, as the free Hamiltonian $h_{0}$ is negligible compared to $v$. However, this is not the case. As shown in Fig. 3, the adiabatic and pseudoadiabatic approximations are rather poor, giving values of $1-O$ of about a few percent. The coherent-state and symmetrized approximations are very good for $A=0.015$, but then they become worse for smaller values of $A$. Numerical calculations are very difficult when $S$ is large, because a large oscillator basis is needed. An analytic solution of this limiting case would be desirable.

The interest of this limit case $(A \rightarrow 0, A S$ constant) is not only formal. In nuclear physics there are cases in which weakly bound nuclei interact strongly with targets during a very short time, so that the quantum state is significantly distorted. The validity of the adiabatic approximation in these situations is open to debate [12].

Note that in the definition of the overlap we allow for an overall phase difference between the exact and approximate wave functions. This overall phase difference does not affect any observable. We find that the best approximate calculations (coherent state and symmetrized) only reproduce accurately the phase of the exact wave function when both $A$ and $S$ are small. We think that this is related to the effect of higher-order terms in the commutator series of the evolution operator, which seem to affect only a global phase in the wave function.

So we see from these approximations that a crucial feature of them is the fact that the most relevant terms in the evolution operator conserve the spin projection along the local direction of the magnetic field. This is the basis of the semiclassical calculation performed in [7], in which the states with definite spin projections along the local magnetic field were taken as coherent internal states, and hence their motion could be described in terms of trajectories.

Despite the fact that the approximations discussed here, especially the coherent-state and symmetrized approximations, are very accurate, they do not describe an important effect of the exact evolution operator. In all the approaches described here, the scattering amplitudes for given spin projections along the $y$ axis (the beam axis) are equal, up to a phase factor, to the amplitudes in which the spin projections are reversed. This is a result of the fact that only terms which commute with $I_{B}$ are allowed in the expansion of the evolution operator.

\section{REEXAMINING THE STERN-GERLACH EXPERIMENTS}

In the textbook description of the Stern-Gerlach experiment, the deflection of the beam gives information of the spin projection along the $z$ axis, which is the one that points along the magnetic field at the center of the beam. The deflection of the beam is not sensitive to the spin components along other directions. If, for a spin-1/2 particle, the initial 
spin points along the $x$ axis, $m_{x}=+1 / 2$, the textbook description would indicate that the pattern of scattered particles would be completely equivalent to that one produced by a mixture of $50 \% m_{z}=+1 / 2$ and $50 \% m_{z}=-1 / 2$ particles. The same would be true for $m_{x}=-1 / 2$. So a Stern-Gerlach experiment is not expected to give any asymmetry between different spin projections perpendicular to the $z$ axis.

To investigate this question, we define the asymmetry for a given axis as the difference in the probabilities of finding the scattered particles in a given position in the $(z, x)$ plane for the two spin projections. Thus, we have

$A_{z}(x, z)=\sum_{m m_{0} m_{0}^{\prime}}\left\langle x, z ; m \mid \Phi(t) ; m_{0}\right\rangle\left\langle x, z ; m \mid \Phi(t) ; m_{0}^{\prime}\right\rangle^{*}\left\langle m_{0}\left|\sigma_{z}\right| m_{0}^{\prime}\right\rangle$,

$$
\begin{aligned}
A_{x}(x, z)= & \sum_{m m_{0} m_{0}^{\prime}}\left\langle x, z ; m \mid \Phi(t) ; m_{0}\right\rangle \\
& \times\left\langle x, z ; m \mid \Phi(t) ; m_{0}^{\prime}\right\rangle^{*}\left\langle m_{0}\left|\sigma_{x}\right| m_{0}^{\prime}\right\rangle, \\
A_{y}(x, z)= & \sum_{m m_{0} m_{0}^{\prime}}\left\langle x, z ; m \mid \Phi(t) ; m_{0}\right\rangle \\
& \times\left\langle x, z ; m \mid \Phi(t) ; m_{0}^{\prime}\right\rangle^{*}\left\langle m_{0}\left|\sigma_{y}\right| m_{0}^{\prime}\right\rangle .
\end{aligned}
$$

Note that, in the standard description of the Stern-Gerlach experiment, the spin projection along the $z$ axis is conserved, and thus the asymmetries $A_{x}$ and $A_{y}$ should vanish at all points. This is not the case. As shown in Fig. 4(b), there is a difference in the pattern of particles scattered depending on the spin projection along the $x$ axis. This effect is found to depend on the inhomogeneity of the magnetic field, which is determined by $z_{0}=B_{0} / B_{1} \sigma$. If $z_{0}$ is large, the inhomogeneity of the magnetic field explored by the beam is small and so is $A_{x}$. This asymmetry can be calculated, with various degrees of accuracy, making use of the approximate treatments discussed here. It can also be calculated with the semiclassical treatment of [7]. The origin of this asymmetry can be understood by arguing that the motion in an inhomogeneous magnetic field conserves the spin projection along the local magnetic field, which has a different direction for the different parts of the wave function. This links with the concept of coherent internal states, which were introduced in Ref. [8].

The calculations in Fig. 4(a) show also that there is an asymmetry $A_{y}$ which means that there is a dependence of the spin projection along the $y$ axis. This is a dynamical effect, which does not appear in the semiclassical description. In fact, in the analytic approximations presented here, the value of $A_{y}$ vanishes after the interaction. Only after allowing for some time of free evolution do nonvanishing values of $A_{y}$ develop. The origin of this asymmetry arises from the term $A S^{2} p_{\beta} I_{y}$ which appears in the double commutator $\left[\left[h_{0}, v\right], v\right]$. The effect of this term can be understood because $p_{\beta}$ is the generator of rotations in the $(x, z)$ plane, around the point $x$ $=0, z=-z_{0}$, where the field vanishes. The effect of this term in the expansion of the evolution operator would generate a rotation in the wave function around the point where the field vanishes, which will be opposite for the different spin pro-

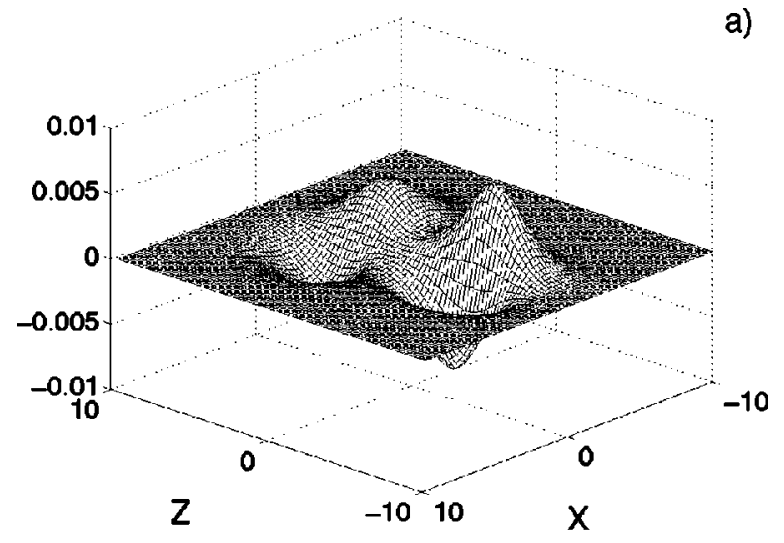

b)

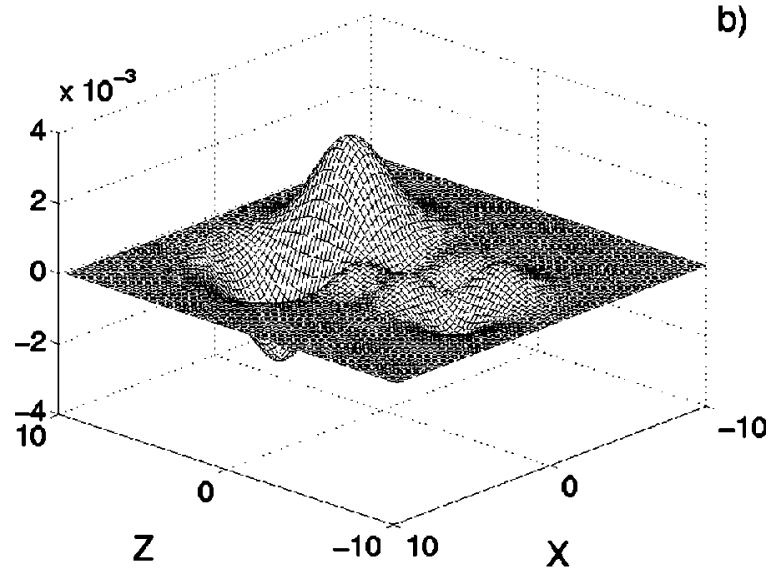

c)

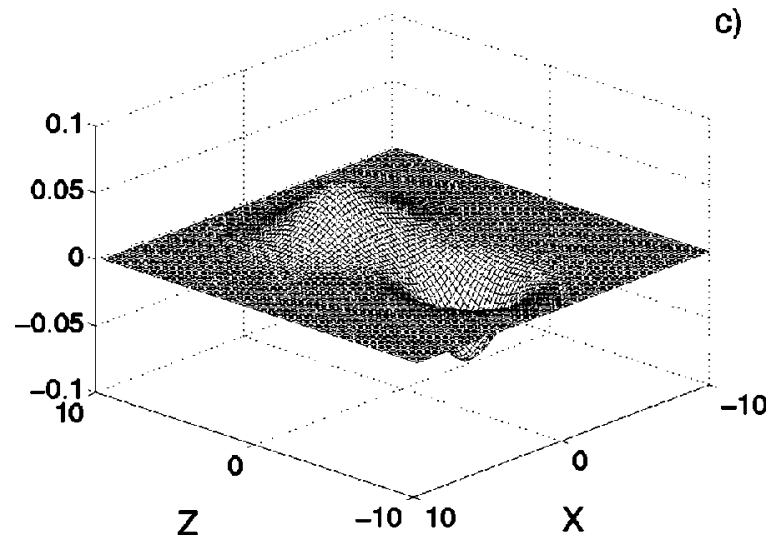

FIG. 4. Asymmetries for particles polarized along the $y$ (a), $x$ (b), and $z$ (c) directions. Note that the maximum asymmetry occurs for particles polarized along the $z$ axis, but that there are important asymmetries for particles polarized along the $x$ and $y$ axes.

jections along the $z$ axis. Indeed, this effect competes with the interaction $v=S \rho I_{B}$, which tends to preserve the spin projection along the direction of the field. The result of this competition is that the magnitude of the asymmetry depends on the ratio $A S / z_{0}$. Note that the asymmetry $A_{y}$ is associated to the dynamically generated term $A S^{2} p_{\beta} I_{y}$. This term depends on the spin projection $I_{y}$ but is independent of $Y$ or $P_{Y}$. So the motion in the $Y$ direction is unaffected by the dynamics, and hence it is given by the free evolution of the $Y$ component of the initial wave packet. 
The fact that all the asymmetries are nonvanishing and also that they have different behavior as a function of $(x, z)$ leads to an exciting possibility. Consider that we have a beam of particles, so that we do not know their polarization state. We can make the beam go through an inhomogeneous field, as described here, and detect the pattern of scattered particles. Let the polarization state be described initially as a density matrix $\rho=1 / 2\left(I+p_{x} \sigma_{x}+p_{y} \sigma_{y}+p_{z} \sigma_{z}\right)$, where $\vec{p}$ is a vector which measures the degree and direction of the beam polarization. Then, the density of particles detected in the $(x, z)$ plane will be proportional to

$$
P(x, z)=P_{0}(x, z)+\frac{1}{2}\left[p_{x} A_{x}(x, z)+p_{y} A_{y}(x, z)+p_{z} A_{z}(x, z)\right] .
$$

This allows us to obtain all the components of the polarization vector from the pattern of scattered particles, when a sufficient number of particles are detected. Note that, in contrast to expression (40), the textbook description of the Stern-Gerlach experiment would be consistent with a probability density given by

$$
\begin{gathered}
P(x, z)=P_{0}(x, z)+\frac{1}{2} p_{z} A_{z}(x, z), \\
A_{z}(x, z)=2 P_{0}(x, z), \quad z>0, \\
A_{z}(x, z)=-2 P_{0}(x, z), \quad z<0 .
\end{gathered}
$$

This expression, when applicable, would allow one to obtain information only on the value of $p_{z}$.

\section{SUMMARY AND CONCLUSIONS}

We have investigated the motion of a particle with spin in an inhomogeneous magnetic field using a quantum mechanical framework. Our aim is to investigate in detail the limitations of the usual textbook approach to Stern-Gerlach experiments, which assumes that the spin projection along the direction of the magnetic field is conserved, while different spin components acquire a momentum which depends on the gradient of the field.

We find that, consistently with a previous semiclassical analysis, there is a sizable probability of spin flip, which depends on the inhomogeneity of the field. Besides, there is a focusing effect in the component that deviates towards the direction in which the modulus of the field decreases. These characteristics are very robust and occur in dynamical situations which are far from the semiclassical limit.

Thus, we can conclude that the Stern-Gerlach experiment is not, even in principle, an ideal experiment, which would "project" the internal state into the eigenvalues of the measurement operator. Moreover, the experiment is not fully reliable, as the positions or momenta of the particles do not give unequivocal information on the spin projection. The magnitude that determines how close a Stern-Gerlach experiment is to an ideal reliable measurement is $z_{0}=\sigma B_{0} / B_{1}$. Only when the magnetic field $B_{0}$ is very large compared to its gradient or when the size of the beam $\sigma$ is very small would the Stern-Gerlach experiment approximate to an ideal reliable measurement.

We have investigated different approximate treatments of the exact quantum mechanical problem. We find that, to a good approximation, the interaction occurs as if the spin projection along the magnetic field at each position was conserved. This indicates that, for each position in the inhomogeneous field, the states with a given spin projection along the magnetic field are coherent internal states. Then, provided that the quantum size of the wave function is small compared to the inhomogeneity of the magnetic field, it is meaningful to approximate the motion of these states in terms of classical trajectories. This justifies the treatment performed in [7].

It is interesting to note that the adiabatic approximation is not accurate, even in the limit of small $A$ (large mass or short interaction time), if, at the same time, the interaction is large so that it generates a fixed deflection angle. This observation can be relevant to cases, such as in nuclear physics [12], in which, although the collision times are short to guarantee the validity of the adiabatic approximation, the forces are so strong to produce a finite deflection.

Our calculations indicate that the Stern-Gerlach experiment is not an ideal measuring apparatus, in the sense of Ref. [5]. However, this does not mean that one cannot acquire accurate knowledge from the spin state of the projectile by observing the statistical results of the experiment. On the contrary, while an idealized Stern-Gerlach experiment will not give any information of the spin projection along the $x$ or $y$ axis, the analysis of a realistic Stern-Gerlach experiment, such as modeled in our calculations, can give the value of all the components of the density matrix that describes the polarization of the beam.

Our analysis supports the idea that the interpretation of realistic experiments does not require the use of the reduction principle, as discussed by several authors in [11]. Thus, the interaction between the spin and magnetic field, which is described in a purely quantum mechanical framework, generates a correlation between the spin polarization of the beam and the final position of the particles of the beam. A measurement of a sufficiently large number of these positions allows one to determine the components of the density matrix of the beam with sufficient statistical accuracy. The reduction principle is not required in this argument.

\section{ACKNOWLEDGMENT}

This work has been partially supported by the Spanish MCyT, Projects No. FPA2002-04181-C04-04 and BFM200203315 . 
[1] R. Eisberg and R. Resnick, Quantum Physics (Wiley, New York, 1974).

[2] J. M. Levy-Leblond and F. Balibar, Quantics (North-Holland, Amsterdam, 1990).

[3] E. Merzbacher, Quantum Mechanics (Wiley, New York, 1998).

[4] A. Messiah, Mechanique Quantique (Dunod, Paris, 1965).

[5] J. von Neumann, Mathematical Foundations of Quantum Mechanics (Princeton University Press, Princeton, 1955).

[6] A. Bassi and G. Ghirardi, Phys. Lett. A 275, 373 (2000).

[7] S. Cruz-Barrios and J. Gómez-Camacho, Phys. Rev. A 63, 012101 (2000).
[8] S. Cruz-Barrios and J. Gómez-Camacho, Nucl. Phys. A 636, 70 (1998).

[9] B. M. Garraway and S. Stenholm, Phys. Rev. A 60, 63 (1999).

[10] H. M. Franca, T. W. Marshall, E. Santos, and E. J. Watson, Phys. Rev. A 46, 2265 (1992).

[11] Quantum Theory Without Reduction, edited by M. Cini and J-M. Levy-Leblond (Adam Hilguer, Bristol, 1990).

[12] R. C. Johnson, in An Advanced Course in Modern Nuclear Physics, edited by J. M. Arias and M. Lozano (SpringerVerlag, Berlin, 2001), pp. 259-291. 